\title{
Initial results using Eddy Current Brakes as Fast Turn-on, Programmable Physical Dampers for Haptic Rendering
}

\author{
Gianni Campion*, Andrew H. Gosline ${ }^{\dagger}$ and Vincent Hayward \\ Haptics Laboratory, Center for Intelligent Machines \\ McGill University, Montréal, Québec, Canada
}

\begin{abstract}
We demonstrate the use of eddy current braking as programmable, fast turn-on, physical damping to improve both the impedance range and stability of a haptic interface while rendering virtual walls and friction.

CR Categories: H.5.2 [Information Interfaces and Presentation]: User Interfaces-Haptic I/O; H.5.1 [Information Interfaces and Presentation]: Multimedia Information Systems-Artificial, augmented, and virtual realities
\end{abstract}

Keywords: Haptic rendering, actuators, haptic devices, stability

\section{INTRODUCTION}

Haptic interface technology has recently begun to receive a great deal of attention from researchers because of the many benefits it brings to science, engineering, and industry. The ability to display the mechanical response of virtual objects through a haptic interface has many applications, such as medical training, exploration, and manufacturing. Naturally, the haptic interface hardware itself plays a large role in the fidelity of the interaction that a user experiences. To date, most available interfaces use only active elements, such as electric motors, to drive linkages or knobs directly or through torque amplification.

Recently, the use of programmable passive elements such as brakes or dampers have been investigated [5]. An and Kwon discuss the use of Magnetorheological (MR) particle brake as programmable dampers for haptic rendering [1]. Also, Mehling et al. shunt a DC motor with a capacitor and resistor in series to create frequency dependent electrical damping [7]. In both works, it is shown that the use of physical damping can improve the stability, and hence impedance range of a haptic interface, but the previously proposed methods for adding damping have limitations. MR particle brakes are programmable, but have long turn-on time [4], and the method proposed by Mehling et al. is not programmable. We propose an alternate method to create damping in a haptic interface that uses eddy current brakes.

\section{EDdy CURRENT BRAKING}

Eddy current brakes are simple magnetic devices that consist of a conductor moving through a magnetic field. When the conductor moves, eddy currents are induced and a resistive force is generated due to dissipation induced by the Joule Effect. At low speeds, when the effects of the magnetic field induced by the eddy currents can be neglected, the resistive force is proportional to the speed of the

\footnotetext{
*e-mail: champ@cim.mcgill.ca

†e-mail: andrewg@cim.mcgill.ca

†e-mail: hayward@cim.mcgill.ca
}

conductor [8]. The physics behind eddy current brakes makes them ideal candidates as programmable dampers for haptics because they are fast, friction free, and linear in the range of velocities in haptic rendering.

\section{Prototype Haptic Display}

A prototype haptic display that incorporates eddy current brakes has been constructed using the Pantograph as a test-bed [2], as shown in Figure 1. An aluminum blade has been added to each base arm of the Pantograph. The blades were designed to minimize inertia, and to rotate concentrically around each motor shaft, ensuring that the brake acts at a constant radius throughout the workspace.
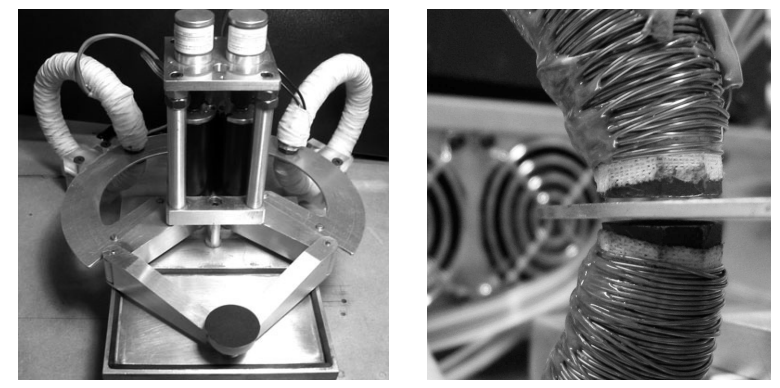

Figure 1: Eddy Current Dampers Attached to each arm of the Pantograph.

Identical toroidal electromagnets were constructed with a core machined out of iron and wrapped with 24 gauge enamel coated wire coils. A thin slot was cut through each core to allow the damper blades to pass through the magnet with a minimal air gap. Each magnet is driven in current mode by a Quanser LCAM connected to a $48 \mathrm{~V}$ power supply. The rise time of this amplifiermagnet combination is $2.6 \mathrm{~ms}$, and the maximum possible damping on a joint is approximately $6 \mathrm{mN} \cdot \mathrm{m} \cdot \mathrm{s}$ peak and $2 \cdot \mathrm{mN} \cdot \mathrm{m} \cdot \mathrm{s}$ continuous.

\section{Experimental Results}

Colgate and Schenkel related stiffness, damping, and update frequency required for the passive simulation of a virtual wall to the amount of physical damping that the device has [3]. From the expression they developed, it is clear that achievable virtual stiffness is a function of the amount of physical damping present in the system. We have performed preliminary experiments with virtual walls and shown that the introduction programmable damping indeed does stabilize a wall that would otherwise be active. Figure 2 shows the results from experiments with wall stiffnesses of $400 \mathrm{~N} \cdot \mathrm{m}^{-1}$ and $1200 \mathrm{~N} \cdot \mathrm{m}^{-1}$. These experiments were performed using a pretensioned elastic band to thrust the end effector into the virtual wall, and to hold it up against the wall in a reproducible manner. A horizontal unidirectional linear spring was used to render the vertical 


\section{4th Symposium on Haptic Interfaces For Virtual Environment And Teleoperator Systems IEEE Virtual Reality 2006 (IEEE-VR2006)}

virtual wall. In the physically damped wall algorithm, the dampers were only turned on when the end effector was inside the wall.

In Figure 2, the plot lines changes thickness at $t=2 \mathrm{~s}$, that is, when the command is given to the electromagnets. It is clear that once the system receives physical damping, the limit cycle present in the $400 \mathrm{~N} \cdot \mathrm{m}^{-1}$ case is quenched, while the limit cycle in the $1200 \mathrm{~N} \cdot \mathrm{m}^{-1}$ is reduced in magnitude.
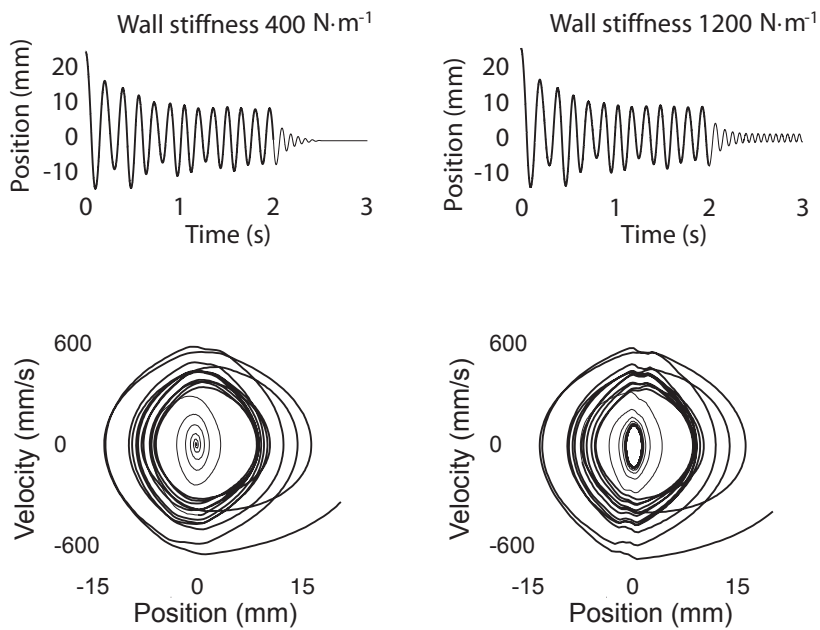

Figure 2: Experiment with Physically Damped Virtual Walls (Dampers on for time $>2 s$ )

In addition, we have added programmable damping to the stuck region of the friction model described by Hayward and Armstrong [6]. This model simulates the stuck region as a linear elastic spring, hence, in the absence of dissipation in the device, the model is prone to limit cycles when stuck. The addition of physical damping alleviated these limit cycles allowing this region to become small without affecting stability.

\section{Conclusions AND Future Work}

A prototype haptic interface with eddy current dampers has been designed and built. The benefits of programmable eddy current damping in haptic rendering have been investigated with preliminary virtual wall and virtual friction experiments. Despite the encouraging preliminary results, much future work is needed. Firstly, the damper blades add considerable inertia to the device, and future design iterations will attempt optimize the trade-off between inertia and damping torque. Secondly, because the rise time of an electromagnet is simply a function of its inductance, it should be relatively easy to achieve a faster turn-on time with amplifiers that can deliver a higher driving voltage than the ones used in this work. Thirdly, it is important to determine the characteristics of the damping provided by eddy current brakes experimentally so that they can be better used in future control/passivity observer schemes.

\section{ACKNOWLEDgments}

This work was supported by a Collaborative Research and Development Grant from the Natural Sciences and Engineering Research Council of Canada (NSERC) "High Fidelity Surgical Simulation".

\section{REFERENCES}

[1] J. An and D.S. Kwon. In haptics, the influence of the controllable physical damping on stability and performance. In Proc. IROS 2004 IEEE/RSJ Int. Conf. Intelligent Robots and Systems, pages 1204-1209, 2004.

[2] G. Campion, Q. Wang, and V. Hayward. The Pantograph Mk-II: A haptic instrument. In Proc. IROS 2005, IEEE/RSJ Int. Conf. Intelligent Robots and Systems, pages 723-728, 2005.

[3] J. E. Colgate and G. Schenkel. Passivity of a class of sampled-data systems: Application to haptic interfaces. In Proceedings of American Conference on Control, pages 3236-3240, 1994

[4] M. Gogola and M. Goldfarb. Design of a PZT-actuated proportional drum brake. IEEE Transactions on Mechatronics", 4(4):409-416, 1999.

[5] D. Grant. Two new commercial haptic rotary controllers. In Proc Eurohaptics, page 451, 2004

[6] V. Hayward and B. Armstrong. A new computational model of friction applied to haptic rendering. In P. Corke and J. Trevelyan (Eds), editors, Experimental Robotics VI, Lecture Notes in Control and Information Sciences, volume 250, pages 403-412. Springer Verlag, Heidelberg, Germany, 2000.

[7] J. S. Mehling, J. E. Colgate, and M. A. Peshkin. Increasing the impedance range of a haptic display by adding electrical damping. In Proc. First Joint Eurohaptics Conference and Symposium on Haptic Interfaces for Virtual Environments and Teleoperator Systems WHC'05, pages 257-262, 2005.

[8] H. D. Wiederick, H. Gauthier, D. A. Campbell, and P. Rochon. Magnetic braking: Simple theory and experiment. American Journal of Physics, 55(6):500-503, 1987. 